\title{
Planeación financiera para el retiro desde la perspectiva de jóvenes universitarios
}

\author{
Financial planning for retirement from the \\ perspective of young university students
}

Quetzalli Atlatenco Ibarra ${ }^{1}$, María Teresa de la Garza Carranza ${ }^{2}$ y Eugenio Guzmán Soria ${ }^{3}$

Fecha de recepción: 27 de septiembre de 2020

Fecha de aceptación: 15 de enero de 2021

\footnotetext{
1- Nacionalidad: Mexicana. Grado: Doctorado en Ciencias de las Administración.Adscripción: Universidad de Guanajuato. (DORCID: https://orcid.org/0000-0001-7352-863X. Correo electrónico: qatlatenco@yahoo.com.mx

2- Nacionalidad: Mexicana. Grado: Doctorado en Ciencias Administrativas. Adscripción: Tecnológico Nacional de México en Celaya. (DORCID: https://orcid.org/0000-0002-4877-3403. Correo electrónico: teresa.garza@itcelaya.edu.mx

3- Nacionalidad: Mexicana. Grado: Doctorado en Ciencias en Economía. Adscripción: Tecnológico Nacional de México en Celaya. DORCID: https://orcid.org/0000-0003-4713-7154. Correo electrónico: eugenio.guzman@itcelaya.edu.mx 


\section{Resumen}

Los jóvenes de hoy en día tendrán que afrontar mayores retos que sus antecesores en lo que respecta al financiamiento de su seguridad económica en la vejez. El objetivo de este trabajo es establecer la relación, desde la perspectiva de jóvenes universitarios, entre sus expectativas de planeación financiera para el retiro y las actitudes y creencias que las propician. Se empleó el método de ecuaciones estructurales para confirmar la validez del constructo y la relación entre las variables. El estudio se desarrolló con una muestra de estudiantes de ingeniería o licenciatura del Tecnológico Nacional de México en Celaya. Se determinó que el conocimiento financiero autopercibido, la perspectiva del futuro y la influencia de los padres impacta de forma significativa, directa y positiva en las expectativas financieras para el retiro.

Palabras clave: pensiones por jubilación, planificación financiera, estudiantes universitarios.

\section{Abstract}

Young people today will face greater challenges than their predecessors when it comes to financing their economic security in old age. The objective of this work is to establish the relationship, from the perspective of university students, between their expectations of financial planning for retirement and the attitudes and beliefs that promote it. The structural equations method was used to confirm the validity of the construct and the relationship between the variables. The study was developed with a sample of undergraduate students from the National Technological Institute of Mexico in Celaya. Self-perceived financial awareness, future outlook, and parental influence were found to significantly, directly, and positively impact financial expectations for retirement.

Keywords: retirement pensions, financial planning, university students. 


\section{Introducción}

$\mathrm{L}$

os jóvenes de hoy en día tendrán que enfrentar la etapa de vejez con mayores dificultades económicas que las de sus padres. Según la Organización para la Cooperación y el Desarrollo Económico (OCDE, 2012), en la actualidad se vive una edad de oro para las pensiones y los pensionistas porque los trabajadores del presente y del futuro tendrán que ser activos laboralmente por más tiempo para recibir pensiones que, sin embargo, serán proporcionalmente más bajas que las de sus antecesores debido a que gran parte de los modelos de financiamiento son de contribución definida, lo que implica que los riesgos de inversión y longevidad deberán ser asumidos por los propios trabajadores.

Esto se debe a que los sistemas de seguridad social en muchos países del orbe han abandonado los principios que los sustentan, como el principio de solidaridad y el de integralidad. De forma tal que ahora es necesario ahorrar adicionalmente a los sistemas de seguridad social para tener una pensión por jubilación, que permita a las personas tener una calidad de vida similar a la que tenían antes de retirarse.

México no está exento de esa problemática, ya que se modificó estructuralmente el modelo de financiamiento y cálculo del monto de pensiones de los dos sistemas de seguridad social más importantes por el número de asegurados. En particular, las personas que laboran en el sector privado y que empezaron a realizar aportaciones a ese sistema de seguridad social a partir del 1 de julio de 1997, la denominada generación AFORE, deberán enfrentar requisitos de jubilación más estrictos que sus antecesores, además de que el monto de su pensión previsional estará totalmente condicionada a las cantidades que hayan reunido en su cuenta individual para el retiro creada exprofeso para ello, lo que conlleva que todos los riesgos deban ser asumidos por cada uno de los trabajadores.

El citado cambio estructural ha magnificado diversas problemáticas. Una de ellas es que las contribuciones al respectivo ramo de seguridad social son reducidas, razón por la cual los fondos que se reunirán serán muy bajos, además de que no se ha incrementado la edad mínima de retiro aun cuando la esperanza de vida tiende a aumentar debido a la transición demográfica que se atraviesa en este país. Esto último implica que la población tiende a envejecer debido a que las tasas de mortalidad y natalidad pasaron de ser altos y sin control a bajos y controlados. La Organización de las Naciones Unidas (ONU), a través de su división de la población, proyecta que el número de personas de 65 años y más en México pasará del 7.61\% del 2020 al 17\% del total de la población en el año 2050 (ONU, 2019). Esa situación también está reflejada en la tasa de dependencia por vejez, que muestra la relación entre la población de 65 años y más y las personas entre 15 y 64 años, de ser de 11.44 en 2020 pasará a ser 26 en 2050, es decir, habrá 26 adultos mayores por cada 100 personas en edad de trabajar (ONU, 2019).

Las pensiones por vejez en México que son más altas son contributivas y se obtienen de los sistemas de seguridad social a través de las ramas obligatorias. Son aproximadamente 8.5 veces mayores en el caso de las mujeres y 11 para los varones, que las pensiones proporcionadas por programas de asistencia social (Puente-Peña, 2018). De acuerdo con el Consejo Nacional de Evaluación de la Política de Desarrollo Social (CONEVAL) el porcentaje de jóvenes, personas entre 15 y 29 años de edad, que realizan aportaciones a algún sistema de seguridad social es del $24.8 \%$ a nivel nacional (CONEVAL, 2018), lo que permite vislumbrar que este grupo etario tendrá enormes dificultades para recibir pensiones suficientes que le permita tener una calidad de vida adecuada en su etapa de adultos mayores. 
Ante ese panorama, uno de los asuntos relacionados con el envejecimiento de la población a atender desde ahora es la seguridad económica en la vejez que Nava-Bolaños et al. (2016) definen como la capacidad de recibir ingresos para satisfacer las necesidades objetivas y subjetivas de una calidad de vida aceptable de dicho grupo etario que les permita conducirse con autonomía, lo que implica independencia para la toma de decisiones. Ni las pensiones por jubilación obtenidas por los sistemas de seguridad social ni las pensiones otorgadas por los programas de asistencia social serán suficientes, por ello es necesario concientizar a los jóvenes de hoy en día acerca de la importancia de prevenir y planear financieramente esa etapa de su vida.

En el contexto mexicano, la investigación que relaciona a los jóvenes y el ahorro para el retiro aun es reciente. Briano-Turrent et al. (2016) inclusive mencionan que los trabajos de investigación reportados en el contexto mexicano y publicados en revistas indizadas están "limitados a estudiantes de educación básica, personal de administración, y personal especializado o laborando en el sector financiero” (p. 4). En un estudio mixto aplicado a jóvenes de entre 15 y 29 años de edad por el Banco Nacional de México y la Universidad Nacional Autónoma de México (BANAMEX y UNAM, 2014), encontraron que poco más de la mitad de los participantes sí tienen el hábito de ahorrar en el corto plazo para comprar algo o salir de viaje y en el largo plazo para comprar una casa.

Estos resultados son coincidentes con los obtenidos por Alvarado-Lagunas y Duana-Ávila (2018) quienes aplicaron una encuesta a estudiantes de nivel medio superior y superior en la ciudad de Monterrey, del estado de Nuevo León en México con la finalidad de cuantificar y pronosticar sus hábitos de ahorro. El retiro laboral no fue significativo en el modelo econométrico aplicado, pero sílos objetivos a corto y mediano plazo. En el mismo estudio elaborado en conjunto por BANAMEX y la UNAM (2014), se asevera que son pocos los jóvenes que ahorran para su vejez (13\%) y esto se debe a su condición laboral. Otra conclusión a la que se llegó en dicho estudio es que consideran que quienes están más preparados para guiarlos en el manejo de su dinero son sus propios padres, las instituciones bancarias y ellos mismos.

La Comisión Nacional de Ahorro para el Retiro (CONSAR, 2017) presentó los resultados de una encuesta cuyo objetivo en parte fue identificar el contexto en el que está inmersa la generación de los jóvenes millennials en cuanto a sus conductas financieras con relación a su retiro laboral. Hallaron que esta generación de jóvenes está más sensibilizada en temas relacionados con su jubilación que generaciones pasadas, pero no realizan acciones concretas para asegurar sus ingresos en la vejez y que están desinformados en cuestiones propias de seguridad social, pues la mayoría considera que la edad para pensionarse es de entre 60 y 64 años.

Esa situación es reforzada por Ordaz-Hernández et al. (2019), quienes realizaron una encuesta centrada en el ahorro para el retiro a estudiantes de los últimos semestres de la Facultad de Contaduría y Administración de la Universidad Veracruzana campus Coatzacoalcos, e identificaron que existe un gran desconocimiento en relación al retiro laboral por parte de los jóvenes, ya que el $41 \%$ considera que las AFORES son del gobierno, además de que desconocen cuáles son las edades de jubilación de acuerdo a las legislaciones de seguridad social (44\%) e inclusive no conocen la definición de seguridad social (72\%).

Cabrera-Ignacio y Zapata-Aguilar (2017), por medio de una encuesta para determinar el nivel de cultura financiera que tienen los estudiantes de la Universidad Tecnológica Metropolitana, hallaron que cerca del $90 \%$ de los jóvenes han considerado ahorrar para su retiro y que, en contraste, solamente el 11\% piensa subsistir en la vejez con apoyos gubernamentales como única fuente de ingresos, lo que implica 
que la mayor parte de los jóvenes están conscientes de la importancia de ahorrar para su retiro. Esto se refuerza, aunque indirectamente, con un hallazgo más de CONSAR (2017) ya que la mayor parte de los jóvenes opinan que el monto de su pensión por jubilación será insuficiente porque alcanzará aproximadamente el 30\% de su salario, que es el porcentaje aproximado que se calcula que alcanzarán las pensiones en algunos años.

Por lo anteriormente expuesto, es pertinente conocer cómo fomentar el ahorro para el retiro en los jóvenes, para ello CONEVAL (2018) sugiere que se promueva la divulgación y educación en materia de pensiones por jubilación como una manera de mejorar la evaluación de las políticas públicas, de forma que redunden en mayor bienestar para la población. Así, el objetivo de la investigación aquí reportada es establecer la relación entre las actitudes y creencias que propician las expectativas de planeación financiera para el retiro mediante el método de ecuaciones estructurales, desde la perspectiva de jóvenes universitarios.

\section{Métodos y materiales}

Esta investigación es cuantitativa, explicativa y transversal debido a que se emplearon técnicas de estadística multivariantes, explica la relación entre variables y se realizó en un punto en el tiempo. Se utilizó el cuestionario elaborado por Koposko et al. (2016) y que fue desarrollado para analizar las creencias, actitudes y comportamientos relacionados con la planeación financiera para el retiro, los ítems que conforman el cuestionario se encuentran en el anexo 1. Ese instrumento fue aplicado en una muestra de estudiantes de la Universidad Autónoma de Yucatán, en México y sus resultados se compararon con las respuestas obtenidas de estudiantes la Oklahoma State University. Dicho instrumento consta de cinco factores medidos a través de 16 ítems con una escala Likert de 7 puntos en la que 1 indica que el encuestado está totalmente en desacuerdo con la actitud o creencia, hasta 7 que significa que el encuestado está totalmente de acuerdo.

El primer factor se denomina "Expectativas de la planeación financiera para el retiro" su finalidad es determinar qué tanto los jóvenes universitarios planean financieramente su periodo de jubilación y en qué medida consideran que lograrán sus objetivos de planeación y ahorro. El segundo factor se ha llamado "El conocimiento financiero autopercibido para la etapa de retiro", con el cual se busca medir el grado en el que los estudiantes califican su propio conocimiento acerca de cómo financiar su etapa de retiro.

El tercer factor fue nombrado "Claridad de las metas financieras en la etapa de retiro", mide la precisión en cuanto a la planeación de asuntos referidos a cómo piensan los encuestados que será su calidad de vida en la etapa de jubilación y cuánto deberán ahorrar para ello, este factor es aún más específico que el anterior por los ítems que lo conforman. El cuarto factor lleva por nombre "Perspectiva del futuro", en esta investigación se utiliza para medir qué tanto los mismos encuestados son proclives a visualizar su vida en el futuro y en qué medida lo han hecho. El quinto y último factor, fue titulado "Influencia de los padres", su objetivo es medir qué tanto consideran los encuestados que sus padres les enseñaron y aconsejaron en su niñez, adolescencia y juventud, con relación a la previsión financiera y el ahorro.

Previamente se comprobó la confiabilidad y validez de este instrumento (Atlatenco et al., 2018) en una muestra compuesta por estudiantes, padres de familia y profesores del Tecnológico de Celaya. 
Por lo anterior, se procedió a aplicar esta encuesta a estudiantes de los últimos cuatro semestres de algunas ingenierías y de la licenciatura en Administración que se imparten en el Tecnológico Nacional de México en Celaya, entre octubre y noviembre de 2018. Cabe señalar que el total de la matrícula de reingreso es de 4,671 estudiantes en el año mencionado (Tecnológico Nacional de México en Celaya, 2019). Se utilizaron Stata 15 y Mplus 7 para realizar los análisis estadísticos respectivos. Se comprobó la validez y confiabilidad del instrumento para la muestra en cuestión y se obtuvieron las correlaciones de los ítems. Posteriormente, se efectuó un análisis factorial comprobatorio y, con ello, se comprobó que la variabilidad de los ítems quedara suficientemente explicada por su factor correspondiente. A continuación, se obtuvieron las medidas de bondad de ajuste del modelo y se comprobaron las hipótesis de investigación a través de la técnica de ecuaciones estructurales.

Con base en el estudio desarrollado por Koposko et al. (2016), se establecieron las siguientes hipótesis:

H1: El conocimiento financiero autopercibido para la etapa de retiro, la claridad de las metas financieras en la etapa de retiro, la perspectiva del futuro y la influencia de los padres impacta directa y positivamente en las expectativas financieras para el retiro.

H2: La claridad de las metas financieras en la etapa de retiro y la influencia de los padres impacta directa y positivamente en el conocimiento financiero autopercibido para la etapa de retiro.

H3: La perspectiva del futuro y la influencia de los padres impacta directa y positivamente en la claridad de las metas financieras en la etapa de retiro.

H4: La influencia de los padres impacta directa y positivamente en la perspectiva del futuro.

\section{Resultados}

Se aplicó la encuesta a alumnos de los últimos semestres de las ingenierías y licenciaturas que son impartidas en el Tecnológico Nacional de México en Celaya, se obtuvieron 468 respuestas válidas. El 51.1\% de las personas que respondieron la encuesta son mujeres y el resto son hombres. La edad mediana es 22.26 años con desviación estándar de 1.31 años. La mediana del semestre que se encontraban estudiando es 7 . En el cuadro 1 se muestra la distribución de los alumnos por ingeniería o licenciatura correspondiente. 
Cuadro 1

Distribución de encuestados por ingeniería o licenciatura

\begin{tabular}{|l|c|c|}
\hline & \# alumnos & $\%$ \\
\hline Licenciatura en Administración & 50 & $10.68 \%$ \\
\hline Ingeniería en Gestión Empresarial & 38 & $8.12 \%$ \\
\hline Ingeniería Industrial & 116 & $24.79 \%$ \\
\hline Ingeniería Química & 98 & $20.94 \%$ \\
\hline Ingeniería Bioquímica & 77 & $16.45 \%$ \\
\hline Ingeniería Mecatrónica & 69 & $14.74 \%$ \\
\hline Ingeniería Ambiental & 20 & $4.27 \%$ \\
\hline Total & $\mathbf{4 6 8}$ & $\mathbf{1 0 0 \%}$ \\
\hline
\end{tabular}

Fuente: Elaboración propia

A continuación, se realizó el cálculo de los coeficientes de correlación de los dieciséis ítems que conforman el instrumento de medición, los resultados se muestran en el cuadro 2, así como la media y la desviación estándar de cada ítem.

Cuadro 2

Correlación de los ítems

\begin{tabular}{|c|c|c|c|c|c|c|c|c|c|c|c|c|c|c|c|c|}
\hline & EPF1 & EPF2 & CF1 & CF2 & CF3 & MF1 & MF2 & MF3 & MF4 & PF1 & PF2 & PF3 & PF4 & IP1 & IP2 & IP3 \\
\hline EPF1 & 1 & & & & & & & & & & & & & & & \\
\hline EPF2 & $0.54 *$ & 1 & & & & & & & & & & & & & & \\
\hline CF1 & $0.15^{*}$ & $0.30 * *$ & 1 & & & & & & & & & & & & & \\
\hline CF2 & $0.15^{* *}$ & $0.24 *$ & $0.78^{* *}$ & 1 & & & & & & & & & & & & \\
\hline CF3 & $0.10 *$ & $0.21 *$ & $0.70 * *$ & $0.76^{* *}$ & 1 & & & & & & & & & & & \\
\hline MF1 & $0.15^{*}$ & $0.23 *$ & $0.61 * *$ & $0.68 * *$ & $0.61 * *$ & 1 & & & & & & & & & & \\
\hline MF2 & $0.13 *$ & $0.16 *$ & $0.43^{* *}$ & $0.45^{* *}$ & $0.43 * *$ & $0.61^{* * *}$ & 1 & & & & & & & & & \\
\hline MF3 & $0.11 *$ & $0.19 *$ & $0.50 * *$ & $0.54^{* *}$ & * $0.50 * *$ & $0.68^{* *}$ & $0.71 *$ & 1 & & & & & & & & \\
\hline MF4 & $0.18 *$ & $0.23 *$ & $0.46^{* *}$ & $0.46^{* *}$ & $0.42 *$ & $0.52^{* *}$ & $0.60 *$ & $0.70^{* * *}$ & 1 & & & & & & & \\
\hline PF1 & $0.35 *$ & $0.33 *$ & $0.21^{* *}$ & $0.16^{* *}$ & $0.18^{*}$ & $0.26 * *$ & $0.33 *$ & $0.38^{*}$ & $0.33 * *$ & 1 & & & & & & \\
\hline PF2 & $0.35 * *$ & $0.35 *$ & $0.19 *$ & $0.18^{* *}$ & $0.17 * *$ & $0.23 * *$ & $0.28 * *$ & $0.27^{*}$ & $0.32 *$ & $0.82 * *$ & 1 & & & & & \\
\hline PF3 & $0.27 * *$ & $0.22 *$ & $0.13 * *$ & 0.07 & 0.06 & $0.10 *$ & $0.15 *$ & $0.13^{* * 1}$ & $0.16 * * 1$ & $0.44 *$ & $0.50 * *$ & 1 & & & & \\
\hline PF4 & $0.45 *$ & $0.33 * *$ & \begin{tabular}{|l|} 
\\
\end{tabular} & $0.09 *$ & 0.06 & $0.18^{* *}$ & $0.20 *$ & $0.18^{*}$ & $0.16^{*}$ & $0.52 * *$ & $0.57 * *$ & $0.46 *$ & 1 & & & \\
\hline IP1 & $0.38 *$ & $0.19 *$ & $0.19 * *$ & $0.21^{* *}$ & $0.13 *$ & $0.20 * *$ & $0.22 *$ & $0.17^{* *}$ & $0.16 * *$ & $0.36 *$ & $0.34^{* * *}$ & $0.21 *$ & $0.38 *$ & 1 & & \\
\hline IP2 & $0.31 *$ & $0.21 *$ & $0.21^{*}$ & $0.26^{* *}$ & $0.20 * *$ & $0.25 *$ & $0.26 *$ & $0.22^{*}$ & $0.22 * * 1$ & $0.32 *$ & $0.33^{* *}$ & $0.15^{*}$ & $0.37 *$ & $0.51^{*}$ & 1 & \\
\hline IP3 & $0.29 *$ & $0.22 *$ & $0.22^{* *}$ & $0.22^{*}$ & $0.14 * *$ & $0.20 * *$ & $0.20 *$ & $0.17^{* *}$ & $0.22^{*}$ & $0.25 * *$ & $0.29 *$ & $0.13^{*}$ & $0.29 *$ & $0.55^{*}$ & $0.69 *$ & 1 \\
\hline Media & 6.38 & 5.72 & 3.43 & 3.35 & 3.04 & 3.88 & 4.60 & 4.06 & 4.28 & 5.78 & 5.74 & 5.56 & 6.26 & 5.54 & 5.75 & 5.74 \\
\hline Desviación estándar & 1.00 & 1.07 & 1.44 & 1.60 & 1.59 & 1.57 & 1.71 & 1.64 & 1.65 & 1.45 & 1.40 & 1.57 & 1.12 & 1.57 & 1.41 & 1.54 \\
\hline
\end{tabular}

Nota: ${ }^{*}$ correlación significativa al $0.05 y^{* *}$ correlación significativa al 0.01

Fuente: elaboración propia con base en salida de Stata 15

Del cuadro 2 se observa que las correlaciones entre las variables son positivas para todos los ítems y significativa para la mayoría de ellos. Adicionalmente se muestran la media y la desviación estándar de cada uno de los ítems.

Se determinó la consistencia interna de cada uno de los factores mediante el coeficiente alfa de Cronbach. También se evaluó la conveniencia de realizar el análisis factorial confirmatorio a través del 
cálculo del índice de adecuación muestral Kaiser Meyer Olkin (KMO) y la prueba de esfericidad de Bartlett. Los resultados se encuentran en el cuadro 3.

\section{Cuadro 3}

Índice de consistencia interna, índice KM y prueba de esfericidad de Bartlett por factor

\begin{tabular}{|l|c|c|c|}
\hline \multicolumn{1}{|c|}{ Factor } & Alfa de Cronbach & Índice KMO & Esfericidad de Bartlett \\
\hline Expectativa planeación financiera (EPF) & 0.70 & 0.50 & $\mathrm{p}<0.001$ \\
\hline Conocimiento financiero (CF) & 0.89 & 0.74 & $\mathrm{p}<0.001$ \\
\hline Claridad metas financieras en etapa de retiro (MF) & 0.87 & 0.81 & $\mathrm{p}<0.001$ \\
\hline Perspectiva del futuro (PF) & 0.82 & 0.73 & $\mathrm{p}<0.001$ \\
\hline Influencia de los padres (IP) & 0.80 & 0.69 & $\mathrm{p}<0.001$ \\
\hline
\end{tabular}

Fuente: Elaboración propia con base en salida de Stata 15

En el cuadro precedente se evidencia que cada uno de los factores tiene una buena consistencia interna porque el coeficiente alfa de Cronbach tiene valores entre 0.70 y 0.89 (Oviedo y Campo-Arias, 2005). Por otro lado, se estudió la conveniencia de realizar un análisis factorial confirmatorio por ello se calcularon el índice Kaiser-Mayer-Olkin (índice KMO) y la prueba de esfericidad de Bartlett.

Para todos los factores el índice KMO se encuentra entre 0.69 y 0.81 , a excepción del primer factor que es 0.50 porque, al conformarse por dos ítems, la correlación parcial es igual a la correlación entre los dos ítems. De acuerdo con Zamora-Muñoz et al. (2009), si el índice KMO es mayor que 0.60, el análisis factorial es conveniente porque se muestra que hay una fuerte correlación entre el conjunto de ítems, es decir, entre los ítems que conforman cada dimensión se comparte una porción importante entre ellos.

La prueba de esfericidad de Bartlett arrojó que el valor "p" correspondiente a cada uno de los factores es menor que 0.001 , por lo que se rechaza la hipótesis nula que consiste en que la matriz de correlaciones es una matriz identidad, lo que conllevaría a concluir que las correlaciones no son significativas entre ellas. Por lo anteriormente expuesto, se infiere que es pertinente realizar el análisis factorial ya que la estructura de correlación entre las variables es fuerte para cada factor.

Se comprobó la conveniencia de realizar un análisis factorial confirmatorio (AFC) porque el modelo que se prueba en esta investigación ha sido aplicado en contextos universitarios y fue validado en un entorno universitario local. El AFC se efectúo con el objetivo de determinar las cargas factoriales ya que de esa manera se especifica el coeficiente de correlación entre cada ítem y su respectivo factor, de manera que queda explicada la variabilidad del factor que le corresponde a cada ítem. Conforme a lo indicado por Hair et al. (1999), citados por Zamora-Muñoz et al. (2009), para una muestra mayor de 352, una carga factorial de 0.30 se considera aceptable. Como puede observarse en el cuadro 4 , todas las cargas factoriales son mayores que dicha cantidad. 
Cuadro 4

Cargas factoriales por variable latente

\begin{tabular}{|l|l|l|l|l|l|l|l|l|l|}
\hline \multicolumn{2}{|c|}{ EPF } & \multicolumn{2}{c|}{ CF } & \multicolumn{2}{c|}{ MF } & \multicolumn{2}{c|}{ PF } & \multicolumn{2}{c|}{ IP } \\
\hline EPF1 & 0.716 & CF1 & 0.853 & MF01 & 0.791 & PF01 & 0.884 & IP01 & 0.652 \\
\hline EPF2 & 0.753 & CF2 & 0.920 & MF02 & 0.785 & PF02 & 0.922 & IP02 & 0.829 \\
\hline & & CF3 & 0.827 & MF03 & 0.878 & PF03 & 0.533 & IP03 & 0.830 \\
\hline & & & & MF04 & 0.749 & PF04 & 0.631 & & \\
\hline
\end{tabular}

Fuente: Elaboración propia con base en salida de Mplus 7

Para complementar el análisis de las cargas factoriales, en el cuadro 5 se presentan las comunalidades y la proporción del error. Las comunalidades representan qué proporción de la varianza de cada ítem queda explicada por su factor correspondiente (Fernández-Aráuz, 2015). De manera tal que, por ejemplo, el $51.3 \%$ de la variabilidad del ítem EPF1 y el 56.7\% de la variabilidad del ítem EPF2, se explica por el factor EPF. Mientras que la diferencia de cada uno de esos valores y el 100\% es la proporción de varianza única y representa el porcentaje de la variabilidad que no está explicada por el respectivo factor. En el caso del ítem EPF1 la proporción de varianza única es $48.7 \%$ y del ítem EPF2 es 43.3\%.

Cuadro 5

Comunalidades y varianza única

\begin{tabular}{|c|c|c|c|}
\hline Factor & Ítem & Proporción de la varianza & Varianza única \\
\hline \multirow{3}{*}{ EPF } & EPF1 & 0.513 & 0.487 \\
\cline { 2 - 4 } & EPF2 & 0.567 & 0.433 \\
\hline \multirow{4}{*}{ CF } & CF1 & 0.728 & 0.272 \\
\cline { 2 - 4 } & CF2 & 0.846 & 0.154 \\
\cline { 2 - 4 } & CF3 & 0.684 & 0.316 \\
\hline \multirow{4}{*}{ MF } & MF01 & 0.626 & 0.374 \\
\cline { 2 - 4 } & MF02 & 0.616 & 0.384 \\
\cline { 2 - 4 } & MF03 & 0.771 & 0.229 \\
\cline { 2 - 4 } & MF04 & 0.561 & 0.439 \\
\hline \multirow{4}{*}{ PF } & PF01 & 0.781 & 0.219 \\
\cline { 2 - 4 } & PF02 & 0.850 & 0.150 \\
\cline { 2 - 4 } & PF03 & 0.284 & 0.716 \\
\cline { 2 - 4 } & PF04 & 0.398 & 0.602 \\
\hline \multirow{4}{*}{ IP } & IP01 & 0.425 & 0.575 \\
\cline { 2 - 4 } & IP02 & 0.687 & 0.313 \\
\cline { 2 - 4 } & IP03 & 0.689 & \\
\hline
\end{tabular}

Fuente: Elaboración propia con base en salida de Mplus 7

A continuación, se comprobaron las hipótesis planteadas por medio de la técnica estadística multivariante conocida como ecuaciones estructurales que combina el análisis factorial, el análisis de varianza y el análisis de regresión lineal múltiple en el que se determinan los efectos directos e indirectos de las variables dependientes (endógenas) e independientes (exógenas) del modelo (Ortiz y Fernández-Pera, 
2018). Así mismo, se efectuaron pruebas de bondad de ajuste para evaluar el ajuste global del modelo para "determinar si las relaciones entre las variables del modelo estimado reflejan adecuadamente las relaciones observadas en los datos" (Weston y Gore, 2006, citados por Medrano y Muñoz-Navarro, 2017, p. 227). Los resultados se encuentran en la gráfica 1, en la que se presentan la respectiva gráfica de sendero y las medidas de bondad de ajuste, así como los coeficientes de determinación.

\section{Gráfica 1}

\section{Senderos y medidas de bondad de ajuste}

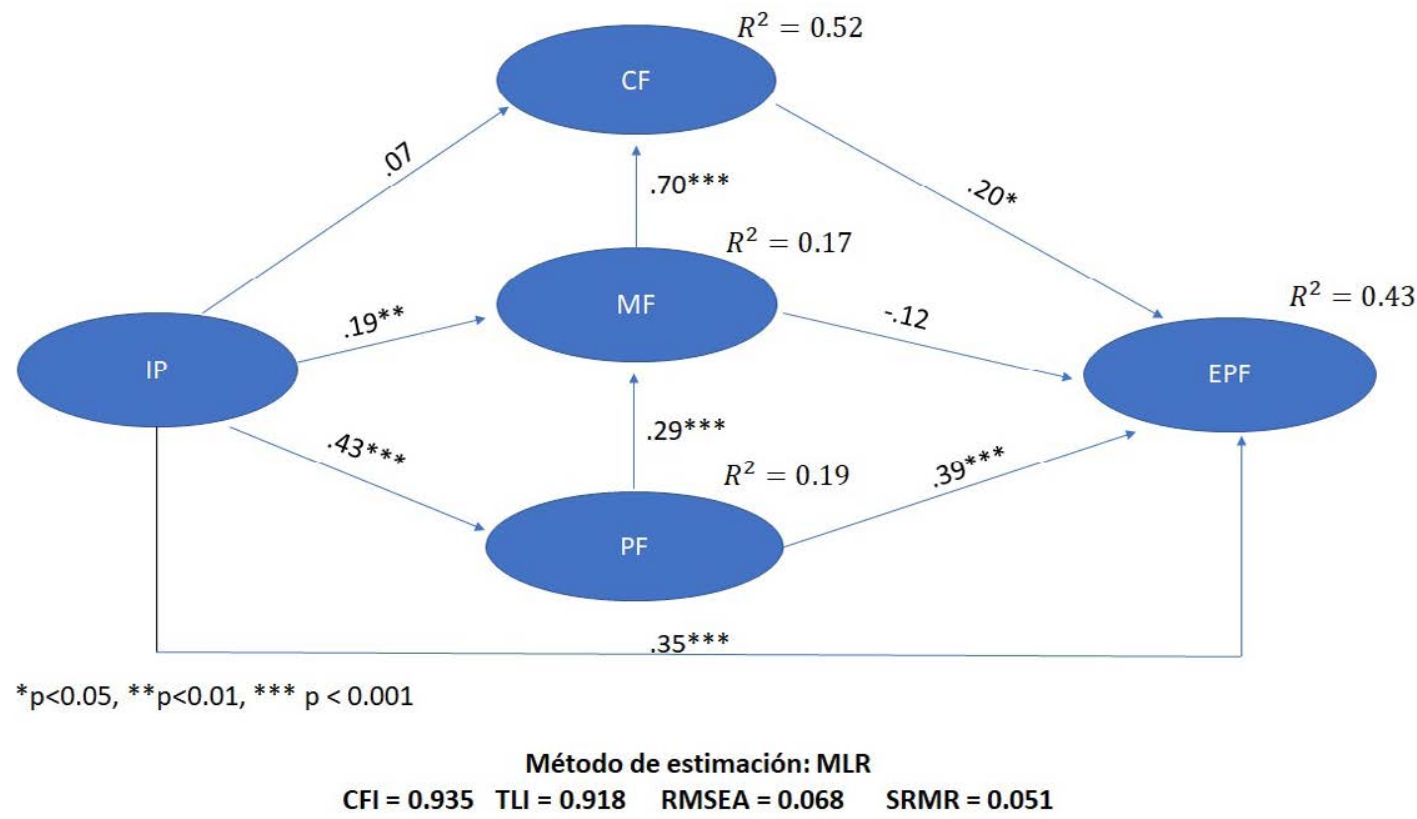

Fuente: elaboración propia

El modelo presenta un ajuste adecuado medido a través de cuatro índices. El índice de ajuste comparativo (CFI) en este modelo es de 0.935 y el índice Tucker Lewis (TLI) es 0.918 , al ser valores superiores a 0.9 se infiere que el modelo se ajusta satisfactoriamente a la estructura teórica y los datos empíricos (Manzano-Patiño, 2017). Mientras que el índice de la aproximación de la raíz cuadrada del error cuadrático medio (RMSEA) fue de 0.068, lo que se considera aceptable, puesto que ese valor está incluido en el intervalo 0.06 y 0.08, (Medrano y Muñoz-Navarro, 2017). También se calculó la raíz cuadrada del residuo estandarizado medio (SRMR), cuyo valor para este modelo fue 0.051 , valor que se considera un indicador de un buen ajuste de acuerdo con Miranda-Zapata et al. (2014).

Para la estimación del modelo estructural se utilizó el método estimación de máxima verosimilitud robusto, debido a que se adapta a los datos que presentan ligeras desviaciones a la distribución normal. Se encontró que existe un impacto directo y positivo del conocimiento financiero autopercibido para la etapa de retiro, la perspectiva del futuro y la influencia de los padres en las expectativas financieras para el retiro. El coeficiente de determinación es del 43\%, lo que implica que las expectativas financieras para el retiro quedan predichas en $43 \%$ por los factores mencionados a excepción de la claridad de las metas 
financieras para el retiro, cuyo impacto no es positivo ni significativo. Esto podría deberse a que dicho factor es aún más específico que el que explica del conocimiento financiero autopercibido para la etapa de retiro, y como se halló en la encuesta nacional ¿Qué piensan los millennial mexicanos del ahorro para el retiro? (CONSAR, 2017) los jóvenes de hoy en día están más sensibilizados en cuestiones de retiro, pero no realizan acciones concretas para planear ni financiar su retiro laboral.

Se observa que el conocimiento financiero autopercibido para la etapa de retiro es impactado de forma positiva y directa por la claridad de las metas financieras en la etapa de retiro en un 52\%. Se comprobó que la claridad de las metas financieras en la etapa de retiro es impactada de forma positiva y directa por la perspectiva del futuro y la influencia de sus padres en $17 \%$. Finalmente, se verifica que la influencia de los padres impacta de forma directa y positiva en la perspectiva del futuro en un $19 \%$.

\section{Discusión}

Conforme a lo reportado por Cabrera-Ignacio y Zapata-Aguilar (2017), el 90\% de los jóvenes han considerado ahorrar para su retiro, lo que conlleva que están conscientes de la importancia de planear el financiamiento de su pensión, de ahí que las expectativas correspondientes se estudien en este documento. Gran parte de los resultados obtenidos en esta investigación son coincidentes con los hallazgos del estudio realizado por Koposko et al. (2016) con estudiantes de nivel de licenciatura de la Universidad Autónoma de Yucatán. Difieren en el impacto que la influencia de los padres tiene en el conocimiento financiero autopercibido en la etapa de retiro, ya que en el estudio que aquí se reporta, no fue significativo. Otra diferencia relevante es el impacto que la claridad de las metas financieras en la etapa de retiro tiene en las expectativas en la planeación financiera, ya que en este trabajo resultaron negativas, pero, además no significativas, lo cuál podría ser un muy importante indicio de un área en la que las escuelas podría intervenir para desarrollar estrategias que deriven en que los jóvenes universitarios visualicen adecuadamente cómo financiar su etapa de retiro laboral y qué esperar de ello.

Los ítems que conforman el factor claridad de las metas financieras en la etapa de retiro hacen referencia a la precisión que los jóvenes manifiestan tener acerca de la información que requieren sobre la jubilación, la calidad de vida visualizada por ellos mismos en esa etapa y cuánto dinero requieren ahorrar para cumplirlo. Al no tener una relación positiva y directa con las expectativas de la planeación financiera para el retiro, se intuye que los jóvenes no vislumbran con precisión cómo vivirán su vejez, qué información es indispensable ni cuánto dinero requerirán para cumplir esa meta, lo que refuerza los hallazgos de Ordaz-Hernández et al. (2019) y CONSAR (2017) en los que se evidencia desconocimiento de los jóvenes en cuanto a edades de retiro y, en general, de la cantidad aproximada de dinero que se requiere ahorrar para obtener pensiones por jubilación suficientes.

Por otro lado, se observa que la influencia de los padres es muy importante en las expectativas y la mayoría de las actitudes de los jóvenes universitarios que respondieron esta encuesta en cuanto su futuro financiero en la etapa de retiro, lo cual es coincidente con el estudio mixto realizado por BANAMEX y la UNAM (2014) en el que los jóvenes estiman que quienes están más preparados para orientarlos en cuestiones financieros son sus propios padres. 


\section{Conclusiones}

En México, como en diversos países del orbe, el financiamiento de las pensiones por jubilación otorgadas a través de los sistemas de seguridad social que recibirán los jóvenes de hoy es un tema crucial que debe atenderse en el presente. Hay diversos aspectos que subyacen en la suficiencia del monto de las pensiones por jubilación, ya que, con el modelo de financiamiento preponderante, todos los riesgos se transfieren a cada trabajador de forma tal que éste debe ser capaz no solo de cumplir con los requisitos de tiempo laborado con aportaciones a la seguridad social, sino también los riesgos financieros y las cantidades relativamente bajas que se contribuyen para el financiamiento de sus propias pensiones.

Los jóvenes universitarios que han iniciado o están iniciando su vida laboral activa, enfrentan un escenario adverso en México debido a que muy pocos reportan estar haciendo aportaciones a las cuentas individuales creadas para financiar las pensiones por jubilación, pero, además, tienen menor acceso a trabajo formal que les permita siquiera acceder a los beneficios de seguridad social, ya que en este país el sistema de seguridad social es ocupacional. Por lo anterior, es necesario conocer qué impacto tienen diversas actitudes y creencias en las metas financieras y en la planificación del retiro de los jóvenes universitarios, para ello se establecieron cuatro hipótesis.

La primera hipótesis se comprobó parcialmente ya que el conocimiento financiero autopercibido para la etapa de retiro, la perspectiva del futuro y la influencia de los padres impacta directa y positivamente en las expectativas financieras para el retiro, aunque la claridad de las metas financieras en la etapa de retiro tiene impacto negativo y no significativo. Se confirmó parcialmente la segunda hipótesis porque la claridad de las metas financieras en la etapa de retiro impacta directa y positivamente en el conocimiento financiero autopercibido para la etapa de retiro, pero el impacto de la influencia de los padres es positivo, aunque no significativo. La tercera hipótesis, en la que se asevera que la perspectiva del futuro y la influencia de los padres impacta directa y positivamente en la claridad de las metas financieras en la etapa de retiro, quedo confirmada. Finalmente, la influencia de los padres impacta directa y positivamente en la perspectiva del futuro que se establece en la cuarta hipótesis, quedó constatada.

El trabajo desarrollado en este documento podría devengar en estrategias que concienticen a los estudiantes en la importancia de la planeación del ahorro para el retiro y del desarrollo de mecanismos que les permitiría realizar esa acción de forma óptima y oportuna. Así mismo, es importante replicar esta investigación y otras relacionadas, en otras regiones y contextos del país para conocer las actitudes y creencias con relación a la planeación financiera para el retiro, de manera que, desde las escuelas, los estudiantes comprendan la problemática que enfrentarán y aprendan como solventarla.

\section{Referencias}

Alvarado-Lagunas, E. y Duana-Ávila, D., (2018). Ahorro, retiro y pensiones: ¿qué piensan los jóvenes adolescentes del ahorro para el retiro? Investigación educativa, 47(122), 1-22.

Atlatenco, Q., De la Garza, M. T. y Guzmán, E., (2019, Mayo 21 - 23). Planeación financiera para el retiro: análisis de la calidad de un cuestionario [Ponencia]. XXIII Congreso internacional de Investigación en Ciencias Administrativas. San Luis Potosí, México. http://acacia.org.mx/wp-content/ 
uploads/2016/10/Acacia-2019_opt.pdf

Banco Nacional de México (BANAMEX) y Universidad Nacional Autónoma de México (UNAM) (2014). Cultura financiera de los jóvenes en México. Sintesis de resultados. BANAMEX. https://www. banamex.com/es/conoce_banamex/quienes_somos/prensa/pdf/book_brujula_digital_2014.pdf

Briano-Turrent, G. C., Quevedo-Monjarás, L. M. y Castañón-Nieto, E. (2016, Junio 27-30). Midiendo la cultura financiera en estudiantes universitarios: El caso de la Facultad de Contaduría y Administración de la UASLP. $1^{\text {a }}$. Reunión internacional de investigación en contabilidad y auditoría (RIICA). Colima, México. https://riica2016.unican.es/wp-content/uploads/2016/06/RIICA-2016-Sesiones-Paralelas-Mesa-5-Comunicaciones.pdf

Cabrera-Ignacio, E. y Zapata-Aguilar, J. A. (2017). Cultura financiera de los estudiantes de la Universidad Tecnológica Metropolitana. Advances in Engineering and Innovation, 2(3), 20-33.

CONEVAL. (2018). Informe de evaluación de política social en México 2018. CONEVAL. https://www. coneval.org.mx/Evaluacion/IEPSM/IEPSM/Documents/IEPDS_2018.pdf

CONSAR. (2017). Encuesta nacional ¿quépiensan los millennials mexicanos del ahorro para el retiro? Secretaría de Hacienda y Crédito Público, CONSAR.https://www.gob.mx/consar/prensa/resultados-dela-encuesta-nacional-que-piensan-los-millennials-mexicanos-del-ahorro-para-el-retiro?idiom=es

Fernández-Araúz, A. (2015). Aplicación del análisis factorial confirmatorio a un modelo de medición del rendimiento académico en lectura. Ciencias económicas, 33(2), 39 - 66. http://dx.doi.org/10.15517/ rce.v33i2.22216

Koposko, J. L., Bojórquez, M. I., Pérez, A. E., y Hershey, D. A. (2016). College student attitudes toward retirement planning: the case of Mexico and the United States. Journal of Personal Finance, 15(2), 52-67.

Manzano-Patiño, A. P. (2017). Introducción a los modelos estructurales. Investigación en educación médica, 7(25), 67-72. https://doi.org/10.1016/j.riem.2017.11.002

Medrano, L. A. y Muñoz-Navarro, R. (2017). Aproximación conceptual y práctica a los modelos de ecuaciones estructurales. Revista digital de investigación en docencia universitaria, 11(1). 219 - 239.

Miranda-Zapata, E., Riquelme-Melia, E., Cifuentes-Cid, H. y Riquelme-Bravo, P. (2014). Análisis factorial confirmatorio de la escala de habilidades sociales en universitarios chilenos. Revista latinoamericana de psicología, 46(2), 73-82.

Nava-Bolaños, I., Ham-Chande, R. y Ramírez-López, B. (2016). Seguridad económica y vejez en México. Revista latinoamericana de población, 10(19), 169-190. https://doi.org/10.31406/relap2016.v10.i2.n19.8

OCDE. (2012). Pension Outlook 2012. OCDE Publishing. https://www.oecd-ilibrary.org/finance-andinvestment/oecd-pensions-outlook-2012_9789264169401-en

ONU. (2019). World Population Prospects 2019. ONU. https://population.un.org/wpp/DataQuery/

Ordaz-Hernández, M. B., Antonio-Pacheco, B., Alonso-Guillén, A. y Gutiérrez-Rodríguez, J. A. (2019). Percepción de la importancia del ahorro para el retiro en los estudiantes de la Universidad Veracruz. Revista internacional La Nueva Organización, 4(11), 129 - 154.

Ortiz, M. y Fernández-Pera, M. (2018). Modelo de ecuaciones estructurales: una guía para ciencias médicas y ciencias de la salud. Terapia psicológica, 36(1), 47-53.

Oviedo, H. C. y Campo-Arias, A. (2005). Aproximación al uso del coeficiente alfa de Cronbach. Revista colombiana de psiquiatría, 34(4), 572-580. 
Puente-Peña, L. F., (2018). Ingresos y gastos del adulto mayor en México: la importancia de las pensiones. Secretaría de Hacienda y Crédito Público, CONSAR. https://www.oissobservatoriovejez.com/publicacion/ingresos-y-gastos-del-adulto-mayor-en-mexico-la-importancia-de-las-pensiones/ Tecnológico Nacional de México en Celaya, (2019). Informe de rendición de cuentas 2018 del Tecnológico Nacional de México en Celaya. Secretaría de Educación Pública, Tecnológico Nacional de México. http://www.itcelaya.edu.mx/irc/IRC_2018.pdf

Zamora-Muñoz, S.; Monroy-Cazorla, L. y Chávez-Álvarez, C. (2009). Análisis factorial: una técnica para evaluar la dimensionalidad de las pruebas. Cuaderno técnico 6. Centro Nacional de Evaluación para la Educación Superior. http://www.ceneval.edu.mx/documents/20182/20789/CuadernoTecnico061aed+\%282\%29.pdf/bd1786a8-3cf0-4cec-bc52-3542201de82c

Anexo 1

Ítems del cuestionario expectativas de la planeación financiera para el retiro

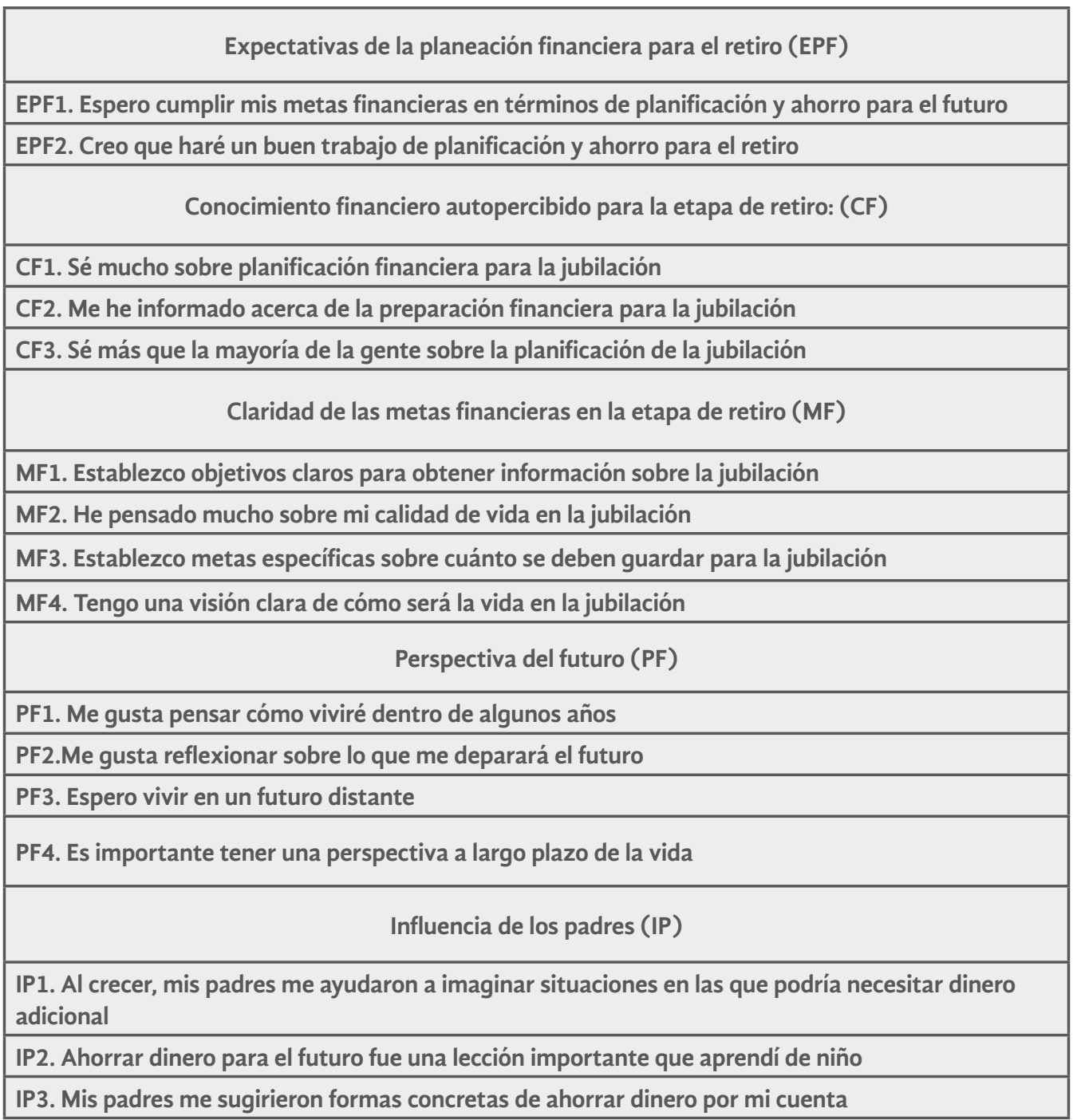

